University of South Carolina

Scholar Commons

Faculty Publications

Electrical Engineering, Department of

$11-1-2003$

\title{
Synergetic Control for DC-DC Boost Converter: Implementation Options
}

\author{
Enrico Santi \\ University of South Carolina - Columbia, esanti00@engr.sc.edu \\ Antonello Monti \\ University of South Carolina - Columbia, monti@engr.sc.edu \\ Donghong Li \\ University of South Carolina - Columbia \\ Karthik Proddutur \\ University of South Carolina - Columbia \\ Roger A. Dougal \\ University of South Carolina - Columbia, dougal@engr.sc.edu
}

Follow this and additional works at: https://scholarcommons.sc.edu/elct_facpub

Part of the Electrical and Computer Engineering Commons

Publication Info

Published in IEEE Transactions on Industry Applications, Volume 39, Issue 6, 2003, pages 1803-1813. http://ieeexplore.ieee.org/xpl/Recentlssue.jsp?punumber=28

(C) 2003 by IEEE

This Conference Proceeding is brought to you by the Electrical Engineering, Department of at Scholar Commons. It has been accepted for inclusion in Faculty Publications by an authorized administrator of Scholar Commons. For more information, please contact digres@mailbox.sc.edu. 


\title{
Synergetic Control for DC-DC Boost Converter: Implementation Options
}

\author{
Enrico Santi, Senior Member, IEEE, Antonello Monti, Senior Member, IEEE, Donghong Li, Student Member, IEEE, \\ Karthik Proddutur, Student Member, IEEE, and Roger A. Dougal, Senior Member, IEEE
}

\begin{abstract}
The theory of synergetic control was introduced in a power electronics context in a previous paper. In this paper, we review the theory, then focus on some practical aspects with reference to both simulations and actual hardware. In particular, we address management of the current limit condition and solve it with several different approaches. Adaptive and other control laws are also introduced to improve the control performance. The various controls are evaluated by applying the classical voltage reference step test and the step load test.
\end{abstract}

Index Terms-Current limit, dynamic parameter adaptation, integral error term, nonlinear control, switching converter, synergetic control theory.

\section{INTRODUCTION}

D ESIGN of controllers for power converter systems presents interesting challenges. In the context of system theory, since power converters are nonlinear time-varying systems, they represent a big challenge for control design.

Much effort has been spent to define small-signal linear approximations of power cells so that classical control theory could be applied to the design. See, for example, [1] and [2]. Those approaches make it possible to use a simple linear controller, e.g., proportional-integral controller, to stabilize the system. The most critical disadvantage is that the so-determined control is suited only for operation near a specific operating point. Further analysis is then necessary to determine the response characteristics under large signal variations [3], [4].

Other design approaches try to overcome the problem by using the intrinsic nonlinear and time-varying nature of switching converters for the control design purpose. A significant example of this approach is sliding mode control, used mostly for continuous-time systems [5]. This control theory has been extensively studied and applied to power electronics systems, since the variable-structure nature of power electronics systems allows a natural application of this theory. The most

Paper IPCSD 03-078, presented at the 2002 Industry Applications Society Annual Meeting, Pittsburgh, PA, October 13-18, and approved for publication in the IEEE TRANSACTIONS ON INDUSTRY APPLICATIONS by the Industrial Power Converter Committee of the IEEE Industry Applications Society. Manuscript submitted for review December 1, 2002 and released for publication July 16, 2003. This work was supported by the U.S. Office of Naval Research (ONR) under Grant N00014-00-1-0131 and Grant N00014-02-1-0623.

E. Santi, A. Monti, D. Li, and R. A. Dougal are with the Department of Electrical Engineering, University of South Carolina, Columbia, SC 29208 USA (e-mail: santi@engr.sc.edu).

K. Proddutur was with the Department of Electrical Engineering, University of South Carolina, Columbia, SC 29208 USA. He is now at 1311 Whaley St., \#807, Columbia, SC 29205 USA.

Digital Object Identifier 10.1109/TIA.2003.818967 important advantages of this approach are order reduction, decoupling design procedure, and insensitivity to parameter changes. Disadvantages are the need of a fairly high bandwidth for the controller, which makes digital control solutions impractical, chattering and variable switching frequency, which introduce undesirable noise in the system.

Another control approach is deadbeat control, which is used for digital systems [6]. Some authors proposed this approach as a way to extend sliding-mode control to discrete-time systems.

In this paper, the focus is on a different approach, synergetic control [7], which tries to overcome the previously described problems of linear control by explicitly using a model of the system for control synthesis. The synergetic control shares with sliding mode control the properties of order reduction and decoupling design procedure, but it has several advantages. First of all, it is well suited for digital control implementation, because it requires a fairly low bandwidth for the controller, but it requires comparatively more complex calculations than sliding mode, which can be easily realized digitally. A second advantage is that it operates at constant switching frequency and it does not have the chattering problems of sliding-mode control, so that it causes less power filtering problems in power electronics applications.

The fact that synergetic control uses a model of the system for control synthesis can be considered both an advantage and a disadvantage. It appears desirable that the control uses all available information on the system for control purposes, but on the other hand it makes the control more sensitive to model and parameter errors. However, as we will demonstrate with experimental results, this problem can be solved.

This paper focuses on the application of synergetic control theory using a boost converter as the example application. In Section II, we review the general synergetic control design procedure. In Section III, this procedure is applied to a boost converter deriving a basic control law. In Section IV, simulation and experimental results for the control under a voltage reference step are presented. In Section V, various improved synergetic control laws are proposed and their performance verified by simulation and by experiment. The first case considered is the introduction of a current limit and two different control laws are proposed. The second case considered is a control law with parameter adaptation. It reduces steady-state output voltage error and provides faster response without increasing the peak inductor current. The third case introduces an integral voltage error term to eliminate the steady-state output voltage error. Finally a control law that does not need an inductor current reference is proposed. In Sections VI and VII, the synergetic 
control is tested under step load variation and the various control laws introduced previously are tested and their performance compared.

\section{Synergetic Control Procedure}

The synergetic control design procedure follows the Analytical Design of Aggregated Regulators (ADAR) method [8]. The main steps of the procedure can be summarized as follows.

Suppose the system to be controlled is described by a set of nonlinear differential equations of the form

$$
\dot{x}=f(x, d, t)
$$

where $x$ is the state vector, $d$ is the control input vector, and $t$ is time.

Start by defining a macro-variable as a function of the state variables

$$
\psi=\psi(x) .
$$

The control will force the system to operate on the manifold $\psi=0$. The designer can select the characteristics of this macro-variable according to the control specifications (e.g., limitation in the control output, and so on). In the trivial case the macro-variable can be a simple linear combination of the state variables.

The same process can be repeated, defining as many macrovariables as control channels.

The desired dynamic evolution of the macro-variables is

$$
T \dot{\psi}+\psi=0, \quad T>0
$$

where $T$ is a design parameter specifying the convergence speed to the manifold specified by the macro-variable. The chain rule of differentiation gives

$$
\dot{\psi}=\frac{d \psi}{d x} \dot{x} .
$$

Combining (1), (3), and (4), we obtain

$$
T \frac{d \psi}{d x} f(x, d, t)+\psi=0 .
$$

Equation (5) is finally used to synthesize the control variable $d$. In the case of a switching converter, control variable $d$ is duty cycle, which is sent to a pulsewidth-modulation (PWM) modulator to create the switch control variable.

Summarizing, each manifold introduces a new constraint on the state space domain and reduces the order of the system.

The procedure summarized here can be easily implemented as a computer program for automatic synthesis of the control law or can be performed by hand for simple systems, such as the boost converter used for this study, which has a small number of state variables.

The synergetic control procedure requires a system model (1) and may be sensitive to system parameter uncertainty. One obvious solution to the sensitivity problem is the adoption of sophisticated observers for parameter determination. This solution is reasonable only if the cost of the control is not a significant concern (e.g., high-power or high-voltage applications). For situations where the control costs are of concern, we will show that

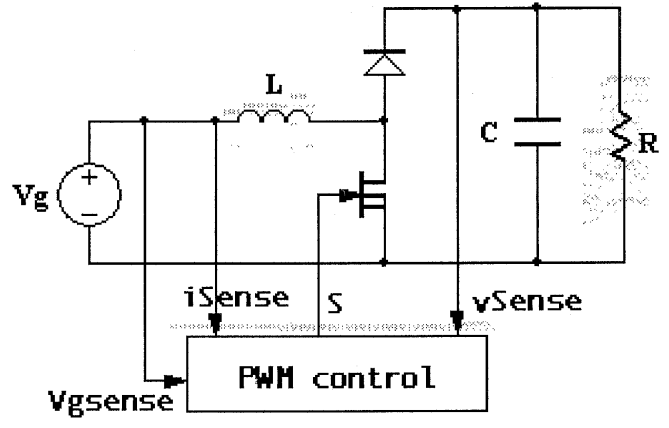

Fig. 1. Boost converter scheme.

suitable selection of the control macro-variables can largely resolve any sensitivity to uncertainty in system parameters. The example of the control of a boost converter will be used to illustrate the issues involved in synergetic control implementation.

\section{Boost Converter Case: An Example of Control SYNTHESIS}

Let us consider the synthesis of a controller for a dc-dc boost converter (see Fig. 1).

The classical averaged model of the converter is

$$
\begin{gathered}
\dot{x}_{1}(t)=-\frac{x_{2}}{L}(1-d)+\frac{1}{L} V_{g} \\
\dot{x}_{2}(t)=\frac{x_{1}}{C}(1-d)-\frac{x_{2}}{R C}, \\
0 \leq d \leq 1
\end{gathered}
$$

where $x_{1}$ is the inductor current, $x_{2}$ the capacitor voltage, and $d$ the duty cycle.

Our objective is to obtain a control law $d\left(x_{1}, x_{2}\right)$ as a function of state coordinates $x_{1}, x_{2}$, which provides the required values of converter output voltage $x_{2}=x_{2 \text { ref }}$ and, therefore, current $x_{1}=x_{1 \text { ref }}$ for various operating modes. The limitation on the duty cycle must be satisfied. We use the procedure described above to solve the problem, i.e., to find $d\left(x_{1}, x_{2}\right)$. The first step is the choice of macro-variable. In general the macro-variable could be any function (including nonlinear functions) of the converter state. For the present time we will limit our investigation to a macro-variable that is a linear function of converter state and has the form

$$
\psi=\left(x_{2}-x_{2 \mathrm{ref}}\right)+k\left(x_{1}-x_{1 \mathrm{ref}}\right) .
$$

Substituting $\psi$ from (7) into (3) yields

$$
T\left(\dot{x}_{2}+k \dot{x}_{1}\right)+\left(x_{2}-x_{2 \mathrm{ref}}\right)+k\left(x_{1}-x_{1 \mathrm{ref}}\right)=0 .
$$

Now, substituting the derivatives $\dot{x}_{1}(t)$ and $\dot{x}_{2}(t)$ from (6) and solving for duty cycle $d$, the following control law is obtained:

$$
d=1-\frac{\left[\frac{k}{L} V_{g}-\frac{x_{2}}{R C}+\frac{x_{2}-x_{2 \mathrm{ref}}}{T}+k \frac{x_{1}-x_{1 \text { ref }}}{T}\right]}{\left[\frac{k}{L} x_{2}-\frac{x_{1}}{C}\right]} .
$$

The expression for $d$ is the control action for the converter controller.

Control law (9) forces the state variable trajectory to satisfy (3). According to this equation, the trajectory converges to manifold $\psi=0$ with a time constant $T$ and then stays on the mani- 


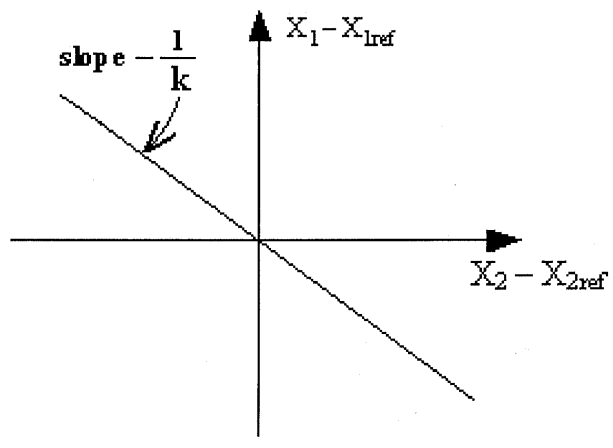

Fig. 2. Geometric interpretation of control law (10) in the phase plane.

fold $\psi=0$ at all times. So, from this point on the state trajectory satisfies

$$
\psi=\left(x_{2}-x_{2 \mathrm{ref}}\right)+k\left(x_{1}-x_{1 \mathrm{ref}}\right)=0 .
$$

This equation establishes a linear dependence between the two state variables $x_{1}$ and $x_{2}$, thereby reducing by one the order of the system. Moving on this manifold, the trajectory eventually converges to the converter steady state: $x_{1}=x_{1 \text { ref }}, x_{2}=$ $x_{2 \text { ref. A geometric interpretation of the control law in the phase }}$ plane is shown in Fig. 2. The steady-state operating point is the origin, where the error goes to zero. Control (10) represents a straight line through the origin with slope $-1 / k$. The system operating point converges to the straight line (the control manifold) and then moves along it to the origin.

We note here that the actual control law used in the experimental verification reported in the following is slightly more complex than this because we desired to account for nonidealities (voltage drops) in the power switches. Therefore, the converter model used to synthesize the control law is a modification of (6) that includes the switch voltage drops. Modeling the conducting MOSFET with its on resistance $R_{s}$ and the conducting diode as a constant voltage source equal to the diode voltage drop $V_{D}$, the averaged state equations describing the boost converter become

$$
\begin{aligned}
& \dot{x}_{1}(t)=-\frac{R_{s}}{L} x_{1} d-\frac{x_{2}}{L}(1-d)+\frac{1}{L} V_{g}-\frac{V_{D}}{L}(1-d) \\
& \dot{x}_{2}(t)=\frac{x_{1}}{C}(1-d)-\frac{x_{2}}{R C}
\end{aligned}
$$

Applying the same procedure described above to these equations, the duty cycle control law becomes

$$
d=1-\frac{\left[\frac{k}{L} V_{g}-\frac{x_{2}}{R C}-\frac{k R_{S}}{L} x_{1}+\frac{x_{2}-x_{2 \mathrm{ref}}}{T}+k \frac{x_{1}-x_{1 \mathrm{ref}}}{T}\right]}{B}
$$

with

$$
B=\frac{k}{L} x_{2}-\frac{x_{1}}{C}-\frac{k R_{s}}{L} x_{1}+\frac{k V_{D}}{L} .
$$

Notice that this equation reduces to (9) for $R_{s}=V_{D}=0$. For simplicity, in the rest of the paper the reported control laws will be derived starting from the ideal state model (6) rather than (11). These equations will be used for the simulations, but the control laws used in the hardware experiments will be based on state model (11).
TABLE I

COMPONENT VALUES FOR LABORATORY PROTOTYPE

\begin{tabular}{l|l}
\hline Rated Input Voltage: $12 \mathrm{~V}$ & Input Inductance: $\mathrm{L}=46 \mu \mathrm{H}$ \\
\hline Rated Output Voltage: $40 \mathrm{~V}$ & $\begin{array}{l}\text { Output Filter Capacitance: } \\
1360 \mu \mathrm{F}\end{array}$ \\
\hline Maximum Load: 100 W & $\begin{array}{l}\text { Nominal load resistance: } \\
\mathrm{R}=35 \Omega\end{array}$ \\
\hline Main Switch: IRF540N & Switching frequency: $50 \mathrm{kHz}$ \\
\hline
\end{tabular}

The control law requires knowledge of boost converter parameters such as inductance $L$, capacitance $C$, and load resistance $R$, and also of state variable reference values. The reference capacitor voltage $x_{2 \text { ref }}$ is known, since it is the desired output voltage. For load resistance $R$ and for the reference inductor current $x_{1 \text { ref }}$, nominal load values are used. If the load is different from the nominal value, there will be some error in the control law, which may cause some steady state error. Some of the improved control laws described later in the paper reduce the effects or completely eliminate these steady-state errors. A load observer may also be used.

\section{System Simulation AND EXPERIMENTAL VERIFICATION}

An extended simulation analysis was conducted to verify the control performance. The simulations were performed both in the Matlab environment and using the VTB simulator [10], [11]. After the theoretical analysis a laboratory prototype was designed and built. The synergetic control is well suited for a digital implementation so a digital-signal-processor (DSP)-based platform was selected for the migration from the VTB environment to the real world. A dSpace DS1103 board was used for the experiments.

The laboratory prototype has the component values listed in Table I.

The dSpace board generates a continuous control signal $d$ between $0-1$ for the duty cycle, which is transformed into a PWM signal by an analog pulse width modulation circuit.

A very good agreement was found between simulation and experiment. As an example, a voltage reference step response from 20 to $40 \mathrm{~V}$ is shown in Fig. 3.

Fig. 4 shows the time evolution of the macro-variable $\psi$. Notice the smaller time scale of $0.5 \mathrm{~ms}$ per division. When the reference step is applied, the state trajectory momentarily leaves the $\psi=0$ manifold, but in approximately $1 \mathrm{~ms}$ goes back to the manifold. This is consistent with first-order (3) with the value of $T=0.3$ ms used: as expected, this transient decays in a time equal to $3 T$. In the time scale of Fig. 3 (10 ms per division), this corresponds to the fast rise of inductor current, which reaches a value of $20 \mathrm{~A}$. Once this transient is over, a second transient follows that satisfies (10). This transient lasts approximately 20 ms. It is usually desirable to choose the time constant $T$ significantly shorter than the response time of the control, so that during most of the transient the system is on the $\psi=0$ manifold.

The following observations can be made regarding the control response waveforms of Figs. 3 and 4. These observations 


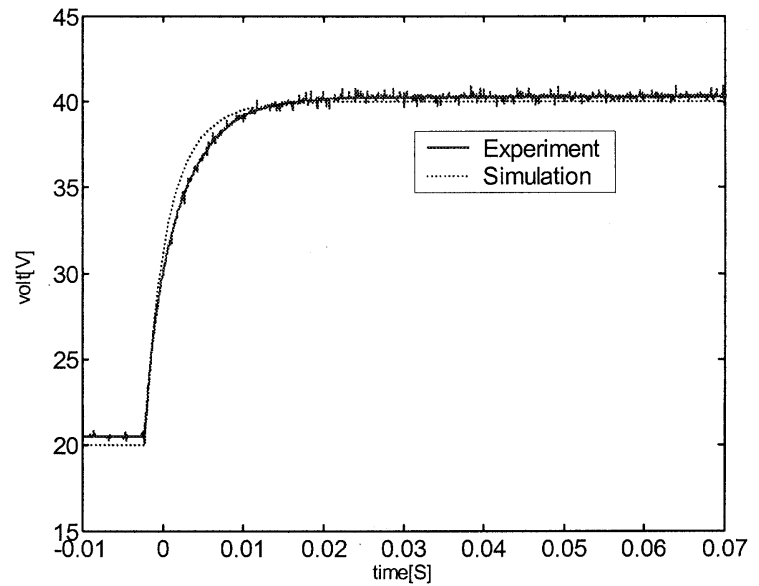

(a)

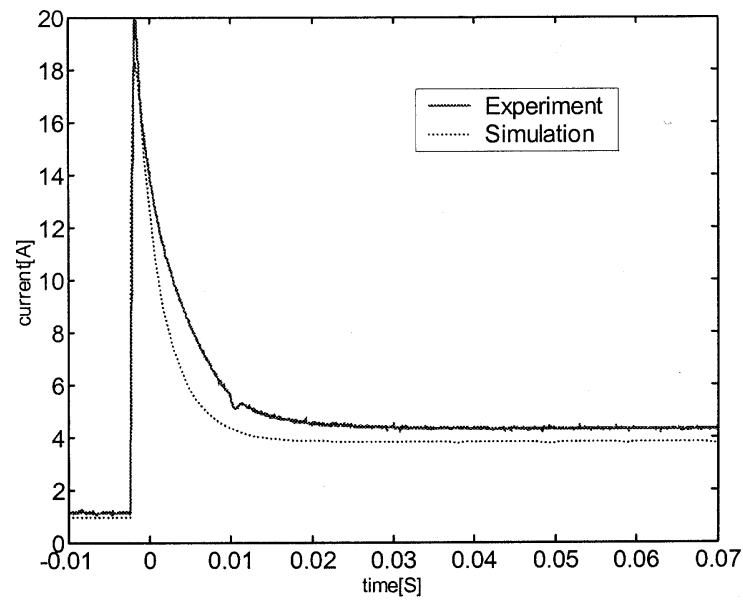

(b)

Fig. 3. Voltage reference step response-simulation and experiment. (a) Output voltage. (b) Inductor current.

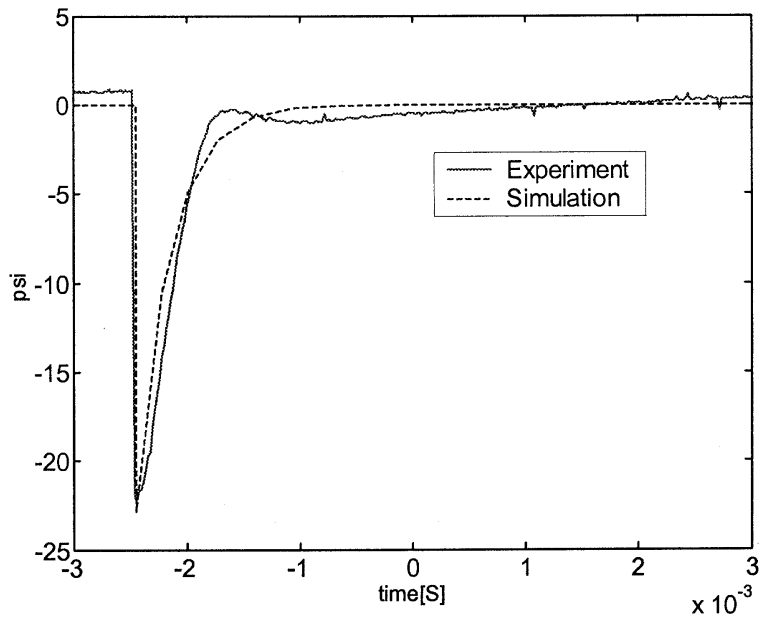

Fig. 4. Voltage reference step response: macro-variable $\psi$-simulation and experiment.

provide motivation for the alternative control laws described in the rest of the paper.

- Current overshoot is not limited. The control law does not limit the inductor current. At startup and during large transients the inductor current can temporarily reach undesir- ably large values. In Section V, two different implementations of a current limit are described.

- Macro-variable $\psi$ has a steady-state value different from zero. The reason for this (see [8]) is that the control law in reality enforces condition (8) and only indirectly condition (10). Therefore, any discrepancy between the real converter parameters and the ones assumed in the control law synthesis may lead to a nonzero value of $\psi$ in steady state.

- A steady-state output voltage error is present. Careful examination of the experimental waveforms shows that a steady-state error is present. Before the step, there is a $0.5-\mathrm{V}$ error and after the step there is a $0.15-\mathrm{V}$ error. In general, this error is caused by small discrepancies between the real converter parameters and the ones used for the control synthesis. The nonzero value of macro-variable $\psi$ is a symptom of the same problem. In order to reduce the error, one approach is to adapt the value of parameter $k$ in the control law (7) because a smaller value of $k$ reduces the voltage error due to the term $k\left(x_{1}-x_{1 \mathrm{ref}}\right)$ in (7). It should be noted that there is an uncertainty in the value of the current reference value $x_{1 \mathrm{ref}}$. Another approach is to add to the control law (7) an integral error term, so that in steady state the error goes to zero.

- The synthesized control variable $d$ given by (9) requires knowledge of converter parameters such as inductance $L$, capacitance $C$ and also load resistance $R$. In particular, load resistance $R$ is needed to calculate the reference inductor current $x_{1 \text { ref }}$ used in the control law (7). Usually, in a switching converter application, it is reasonable to assume that inductance and capacitance values are known, but it is not reasonable to assume that the load characteristics are known or even fixed. So we introduce a modified control that does not use load resistance $R$, but rather uses a high-pass-filtered version of inductor current in (7), so that the control does not need the reference inductor current $x_{1 \text { ref }}$.

- There is a discrepancy in the steady-state inductor current between experiment and simulation, especially when the output voltage is $40 \mathrm{~V}$. This discrepancy is due in part to the fact that the experimental voltage is higher than the simulated voltage as noted above. Another reason is that in the simulation the boost converter model (6) is ideal and, therefore, lossless. In the experiment the losses in the converter will make the inductor current larger than the value predicted by the simulation.

\section{SYNTHESIS OF MODIFIED/IMPROVED CONTROLS}

The previous case illustrated a very simple case of control synthesis that transformed the boost circuit into a first-order system always working on the manifold described by the macrovariable.

Areas of possible improvement were identified above. More complex macro-variable definitions will next be introduced to implement improved control laws. The procedure to derive the new control laws is analogous to the one described above. In the following part of this paper a number of different cases will 


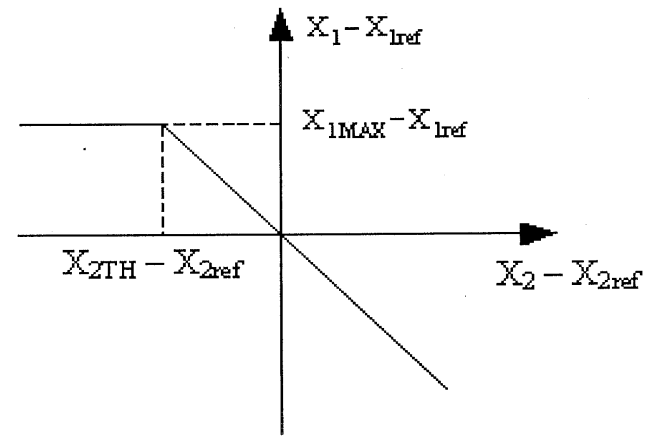

Fig. 5. Piecewise-linear function in the phase plane represents the current limit control law (13) and (14).

be described in detail and their performance evaluated both in simulation and experiment.

\section{A. Current Limit Implementation}

One classical problem relates to imposing a limit on one of the state variables, e.g., limiting the maximum input current. Two different approaches are examined.

In one approach the macro-variable is defined as a piecewiselinear function

$$
\begin{aligned}
& \psi_{1}=(x 2-x 2 \text { ref })+k(x 1-x 1 \text { ref }), \quad \text { for } x_{2} \geq x_{2 T H} \\
& \psi_{1}=x_{1}-x_{1 \mathrm{MAX}}, \quad \text { for } x_{2} \leq x_{2 T H}
\end{aligned}
$$

where $x_{2 T H}$ is the voltage value at which current $x_{1}$ is equal to the limit value $x_{1 \mathrm{MAX}}$. This value can be easily calculated from (10) as

$$
x_{2 T H}=x_{2 \mathrm{ref}}-k\left(x_{1 \mathrm{MAX}}-x_{1 \mathrm{ref}}\right) .
$$

A geometric interpretation of this control law is shown in Fig. 5, which shows the control law (13) and (14) in the phase plane. The steady-state operating point is the origin, where the error goes to zero. Control (13) represents a straight line through the origin with slope $-1 / k$. The current limit given by (14) represents a straight horizontal line.

Repeating the usual synthesis procedure, the control law can be obtained

$$
\begin{aligned}
& d=1-\frac{\left[\frac{k}{L} V_{g}-\frac{x_{2}}{R C}+\frac{x_{2}-x_{2 \mathrm{ref}}}{T}+k \frac{x_{1}-x_{1 \mathrm{ref}}}{T}\right]}{\left[\frac{k}{L} x_{2}-\frac{x_{1}}{C}\right]}, \\
& \quad \text { for } x_{2} \geq x_{2 T H} \\
& d=1-\frac{\left[V_{g}+\frac{L}{T}\left(x_{1}-x_{1 \mathrm{MAX}}\right)\right]}{x_{2}}, \quad \text { for } x_{2}<x_{2 T H} .
\end{aligned}
$$

This approach is easy to implement with a digital controller. It has been simulated and tested and results are shown in Fig. 6. Once again note the good agreement between simulation and experiment.

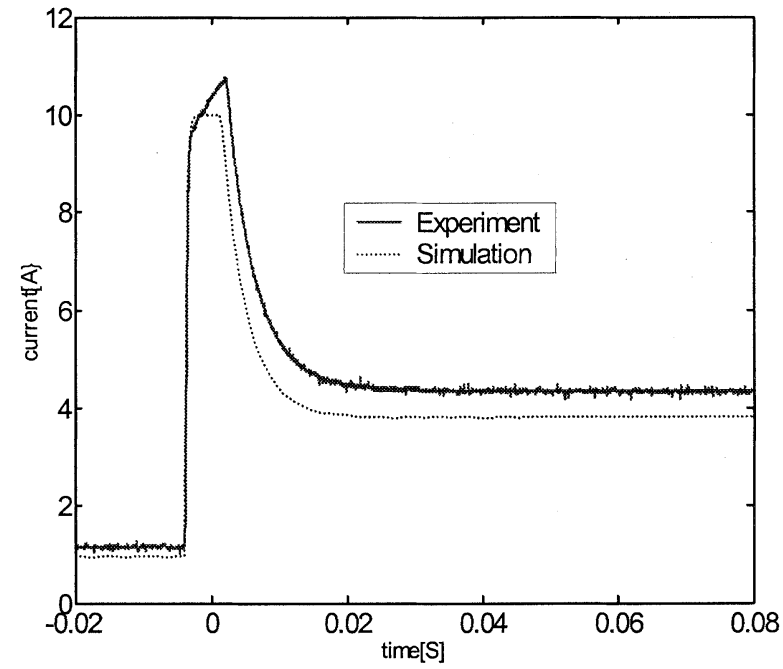

Fig. 6. Current limit using a piecewise-linear function-simulation and experiment.

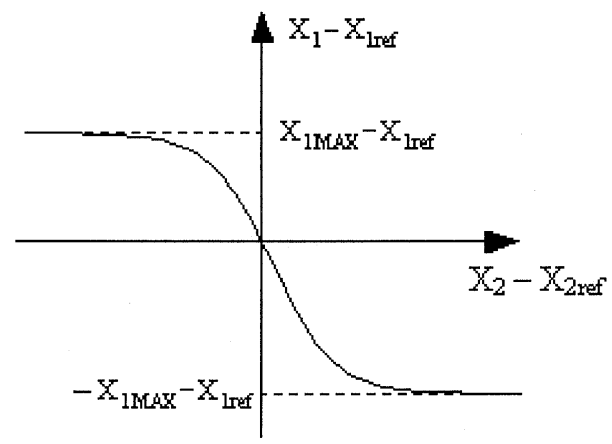

Fig. 7. Geometric interpretation of control law (18).

A second possible approach to implement the current limit is to define a single macro-variable that includes the current limit in its definition. A possible control law is

$$
\psi_{2}=x_{1}+A \cdot \tanh \left[\frac{-x_{1 \mathrm{ref}}+\frac{x_{2}-x_{2 \mathrm{ref}}}{k}}{A}\right]
$$

where $A=x_{1 \mathrm{MAX}}$. This new definition will determine a new manifold where the current $x_{1}$ is naturally limited by the hyperbolic tangent function to the range $\pm A$ whenever the system state is on the manifold. A geometric interpretation of the control law is shown in Fig. 7.

The control law is given by (19), as shown at the bottom of the next page.

The simulation and experiment are shown in Fig. 8. An excellent agreement has been found between simulation and experiment.

\section{B. Control Law With Dynamic Adaptation of Control Parameter}

The choice of the value of control parameter $k$ in the macrovariable definition (7) involves a tradeoff: during transient a relatively large value of $k$ is desirable, because it avoids large overcurrents and excessive stress on the switches. On the other hand, a small value is desirable in steady state, because this reduces 


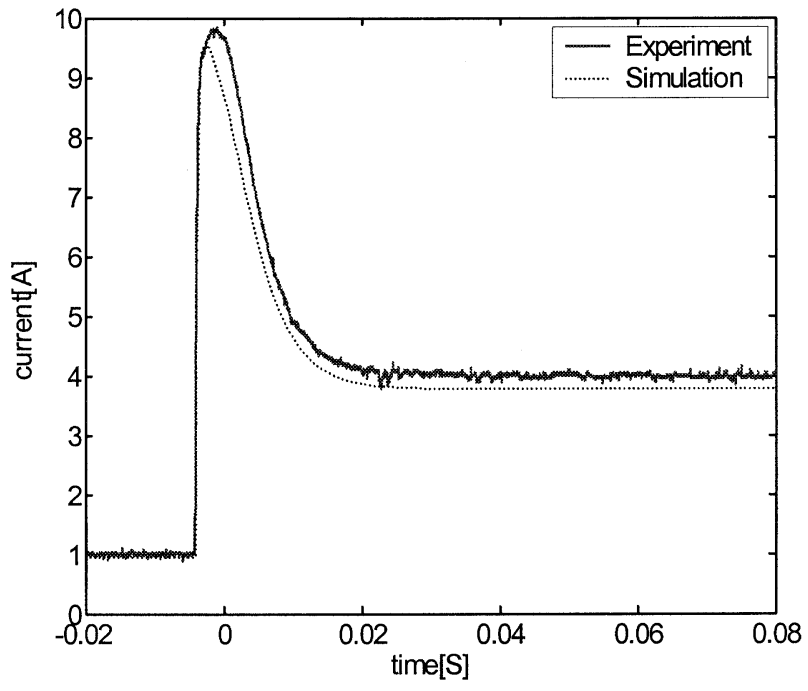

Fig. 8. Current limit with hyperbolic tangent function-simulation and experiment.

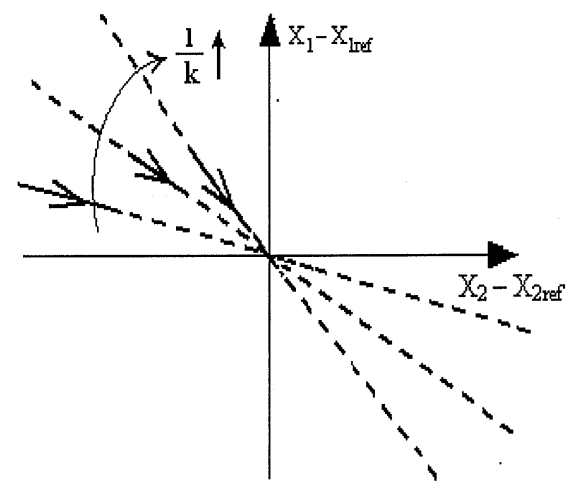

Fig. 9. Geometric interpretation of parameter adaptation (20).

the steady-state output voltage error as explained in [8]. A way around the tradeoff is to dynamically adjust the value of $k$ as a function of output voltage error, reducing $k$ when the output voltage error is small. Based on this consideration, $k$ can be chosen as follows:

$$
k=\alpha+\beta \cdot a b s\left(x_{2}-x_{2 \mathrm{ref}}\right) .
$$

Fig. 9 shows a geometric interpretation of this control law. Far from the origin the error is large and $1 / k$ is small. This situation is represented by the line with smaller slope. As the operating point moves closer to the origin the trajectory slope increases as $1 / k$ increases. The adaptation is a continuous process and the trajectory slope changes continuously. Only three representative lines are shown in the figure.

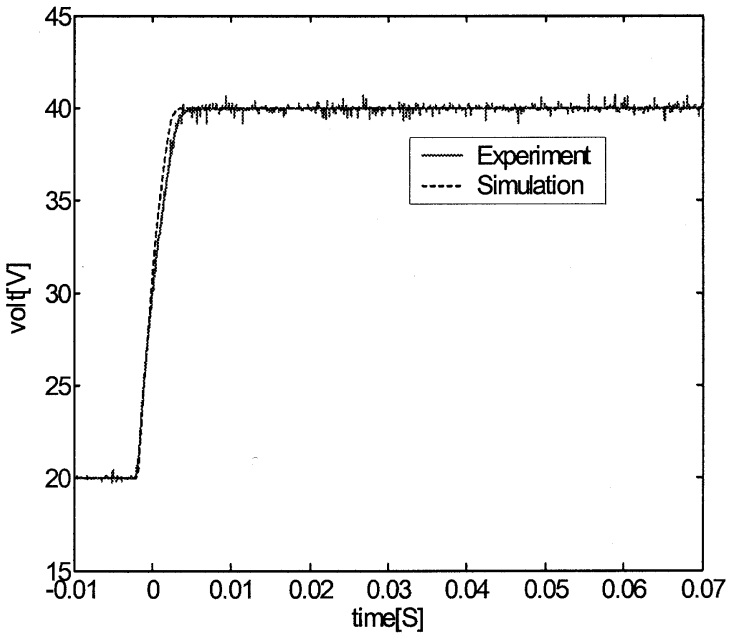

(a)

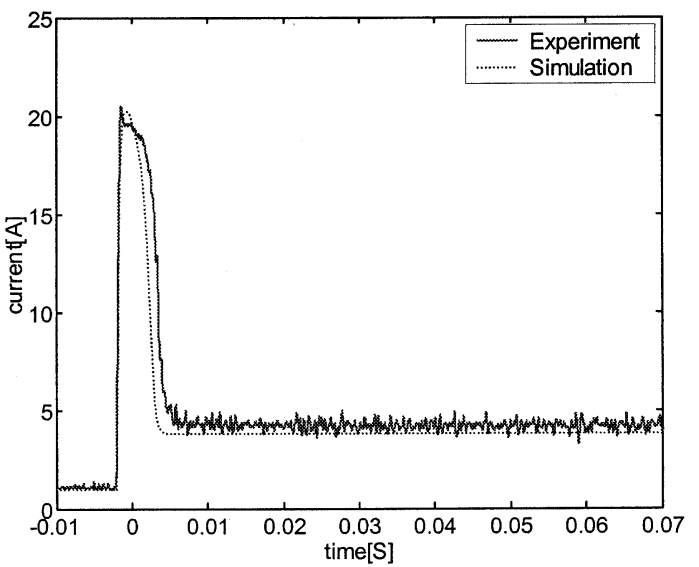

(b)

Fig. 10. Dynamic parameter adaptation. (a) Output voltage. (b) Inductor current.

Using the adapted value of $k$ given by (20) in the control law (7), the simulation and experiment are shown in Fig. 10 for $\alpha=0.03$ and $\beta=0.05$. These results can be compared with those in Fig. 3, where $k=1.0$. First of all, the steady-state error is reduced with respect to Fig. 3. An added benefit is that the final part of the transient, when the output voltage error is small and parameter $k$ is small according to (20), is faster with the modified control law for the same peak inductor current of approximately $20 \mathrm{~A}$.

It is also possible to combine the parameter adaptation with the current limit feature introduced above by using the value of $k$ given by (20) in control law (18) that implements the current limit. The current limit was set at $10 \mathrm{~A}$. The result is shown in

$$
d=1-\frac{\frac{V_{g}}{L}+\frac{x_{1}}{T}-\frac{x_{2}}{k R C \cosh ^{2}\left[\frac{\frac{-x_{1 \mathrm{ref}}+\left(x_{2}-x_{2 \mathrm{ref}}\right)}{k}}{A}\right]}+\frac{A}{T} \tanh \left[\frac{\frac{-x_{1 \mathrm{ref}}+\left(x_{2}-x_{2 \mathrm{ref}}\right)}{k}}{A}\right]}{\frac{x_{2}}{L}-\frac{x_{1}}{k C \cosh ^{2}\left[\frac{-x_{1 \mathrm{ref}}+\left(x_{2}-x_{2 \mathrm{ref}}\right)}{k}\right]}}
$$




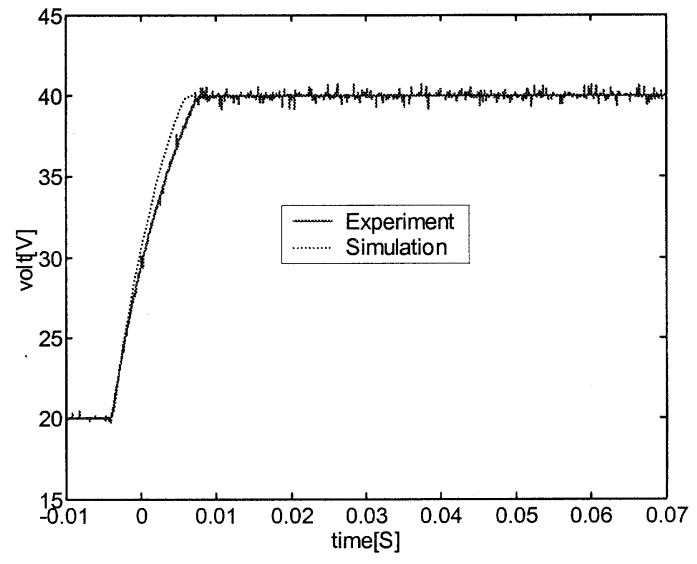

(a)

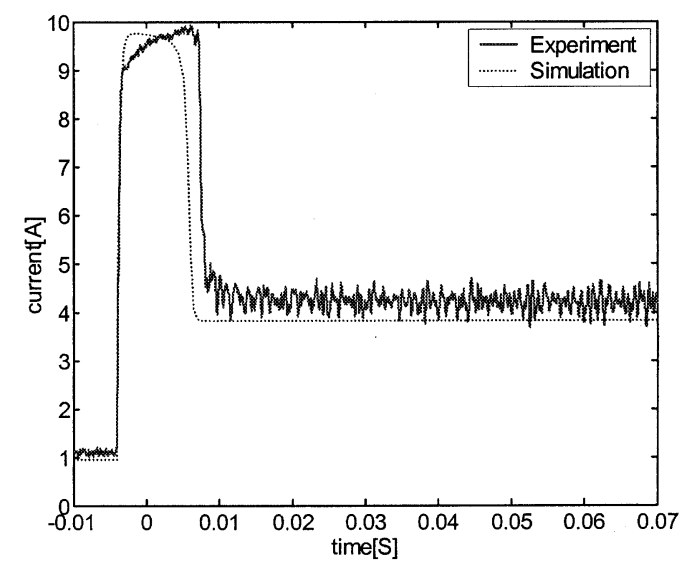

(b)

Fig. 11. Dynamic parameter adaptation with current limit. (a) Output voltage. (b) Inductor current.

Fig. 11. Notice that the inductor current is limited to less than $10 \mathrm{~A}$.

\section{Control Law With Integral Error Term}

In order to eliminate the steady-state error, an integral error term is added to the manifold definition. This term is amplitude limited to avoid windup problem and interference with the synergetic control. The improved macro-variable is

$$
\psi=\left(x_{2}-x_{2 \mathrm{ref}}\right)+k\left(x_{1}-x_{1 \mathrm{ref}}\right)+k_{2} \int\left(x_{2}-x_{2 \mathrm{ref}}\right) d t .
$$

According to the synthesis procedure described in Section II, the control law is derived as (22), shown at the bottom of the page.

Implementing the above control law (22), the simulation and experiments are plotted in Fig. 12. The steady-state error is virtually eliminated, but note the second-order-type behavior with transient overshoot. This is to be expected. The boost converter

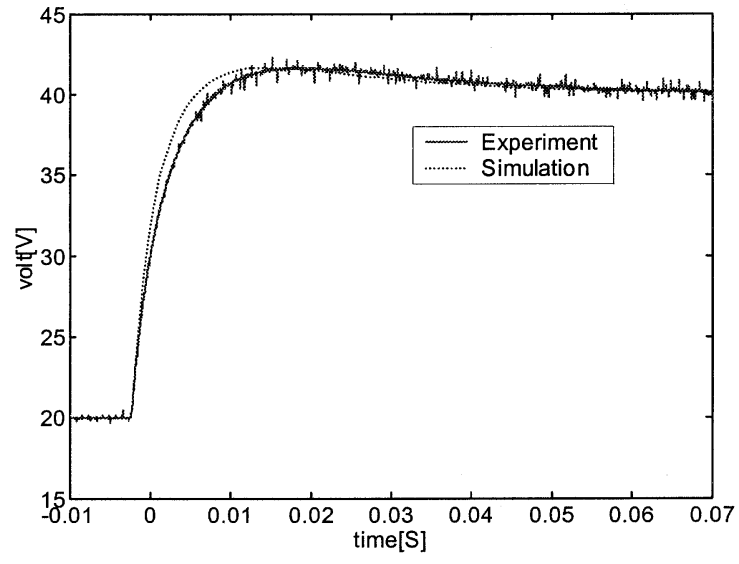

(a)

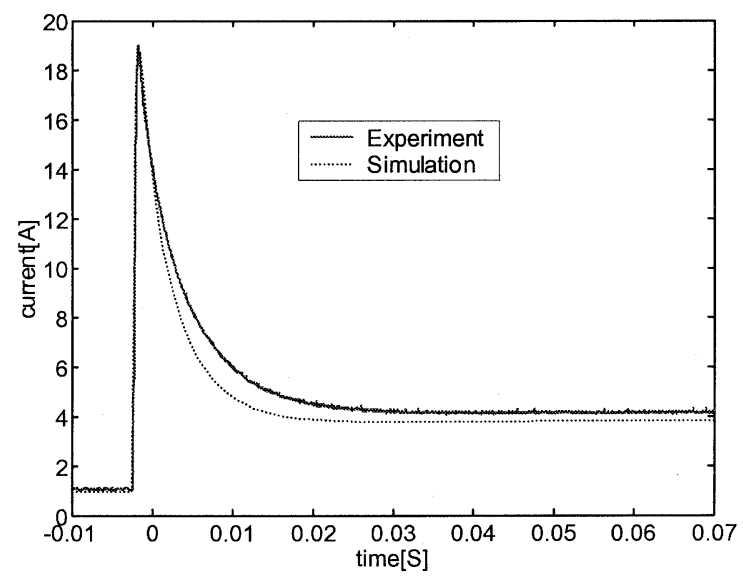

(b)

Fig. 12. Addition of integral error term. (a) Output voltage. (b) Inductor current.

is a second-order system, which becomes first order by virtue of the synergetic control law (10). Notice that the introduction of the integral term increases the system order by one, and returns the controlled system back to second order.

The integral error term can also be added into the current-limited macro-variable (18). The macro-variable becomes

$$
\begin{aligned}
\psi=x_{1}+A \cdot \tanh \left[\frac{-x_{1 \mathrm{ref}}+\frac{x_{2}-x_{2 \mathrm{ref}}}{k}}{A}\right] & \\
& +k_{2} \int\left(x_{2}-x_{2 \mathrm{ref}}\right) d t .
\end{aligned}
$$

Applying the usual synthesis procedure, the control law is obtained as (24), shown at the bottom of the next page, where

$$
y=\frac{-x_{1 \mathrm{ref}}+\frac{x_{2}-x_{2 \mathrm{ref}}}{k}}{A} .
$$

The simulation and experimental results for control law (24) are shown in Fig. 13. The addition of the integral term introduces

$$
d=1-\frac{\frac{k}{L} V_{g}-\frac{x_{2}}{R C}+\frac{x_{2}-x_{2 \mathrm{ref}}}{T}+k \frac{x_{1}-x_{1 \mathrm{ref}}}{T}+k_{2}\left(x_{2}-x_{2 \mathrm{ref}}\right)+\frac{k_{2}}{T} \int\left(x_{2}-x_{2 \mathrm{ref}}\right) d t}{\frac{k}{L} x_{2}-\frac{x_{1}}{C}}
$$




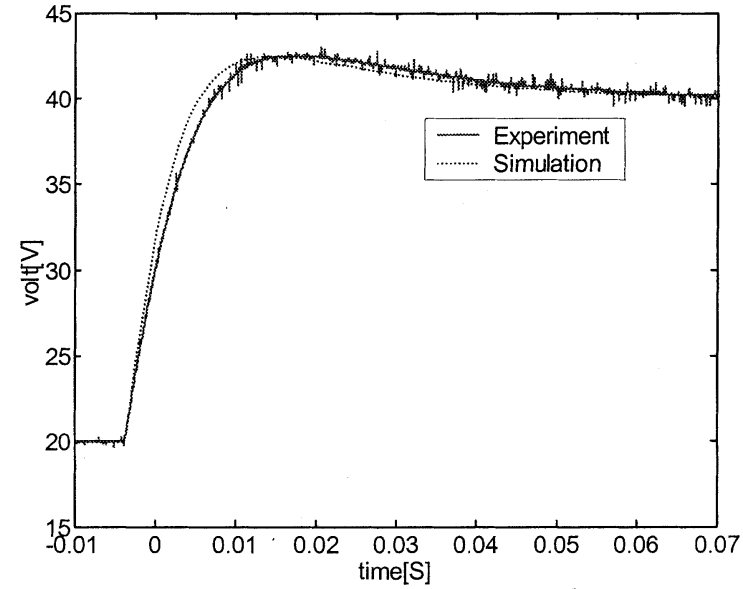

(a)

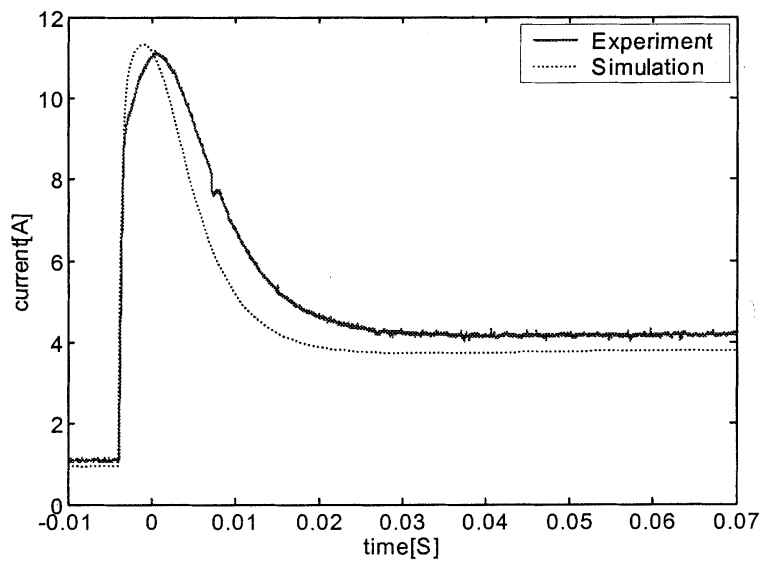

(b)

Fig. 13. Addition of integral error term with current limit. (a) Output voltage. (b) Inductor Current.

a voltage overshoot. It is possible to avoid this by activating the integral error term only when the voltage error is below a certain threshold, so that transient behavior is not affected.

\section{Control Law Using High Pass-Filtered Inductor Current}

An alternative control law can be defined that uses a highpass-filtered measurement of the inductor current in place of the reference current $x_{1 \text { ref }}$ (which, in most applications, is not actually known). The macro-variable becomes

$$
\psi=\left(x_{2}-x_{2 \mathrm{ref}}\right)+k x 1 \frac{s T}{1+s T} .
$$

Comparing the modified macro-variable (25) to the original macro-variable (7), the absence of the current reference value $x_{1 \text { ref }}$ is apparent.

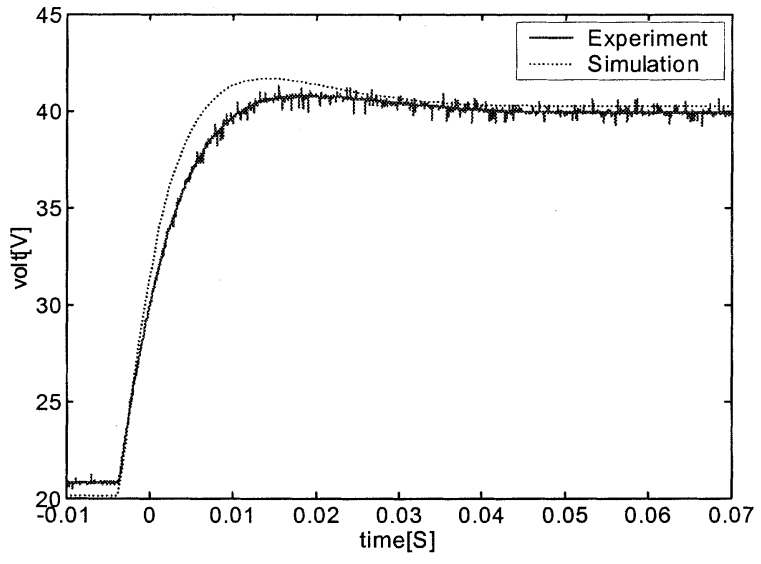

(a)

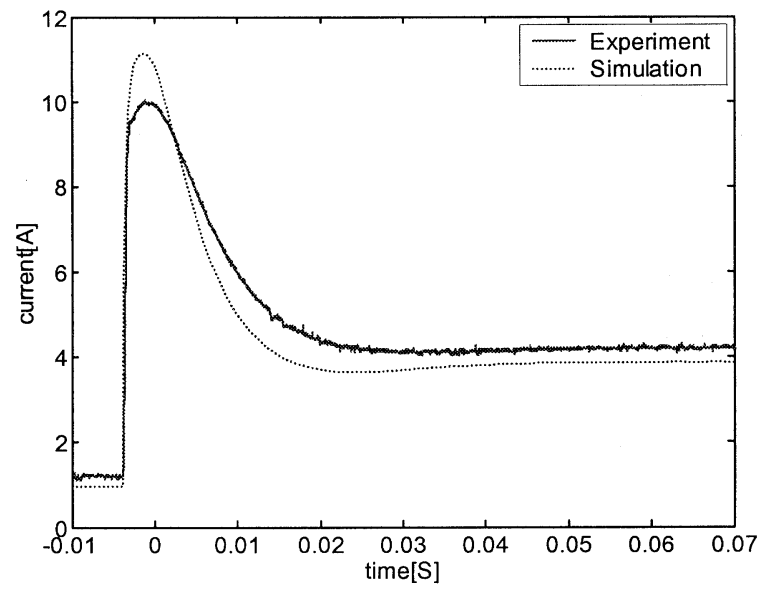

(b)

Fig. 14. High-pass filter, corner frequency $=100 \mathrm{~Hz}$. (a) Output voltage. (b) Inductor current.

The high-pass-filtered inductor current can also be combined with the current limit feature to limit the maximum value of the inductor current.

The simulation and experiments are shown in Fig. 14 with a high-pass-filter corner frequency of $100 \mathrm{~Hz}$.

\section{Simulation AND EXPERIMENTAL RESUlts FOR THE CASE OF STEP-LOAD VARIATION}

Up to this point, the control performance was tested under a reference voltage step. In practice, it is of interest to evaluate the control performance for a step-load variation [12].

The control laws introduced earlier were tested under the condition of a $50 \%$ step change of the load from $35 \Omega$ (nominal load) to $70 \Omega$. The experimental and simulation results for the different control laws both show the startup transient first, followed by the response to step load variation. This was done to verify the large-signal stability of the system under the various

$$
d=1-\frac{\frac{V_{g}}{L}+\frac{x_{1}}{T}-\frac{x_{2}}{k R C \cosh ^{2} y}+\frac{A}{T} \tanh y+k_{2}\left(x_{2}-x_{2 \mathrm{ref}}\right)+k_{2} \int\left(x_{2}-x_{2 \mathrm{ref}}\right) d t}{\frac{x_{2}}{L}-\frac{x_{1}}{k C \cosh ^{2} y}}
$$




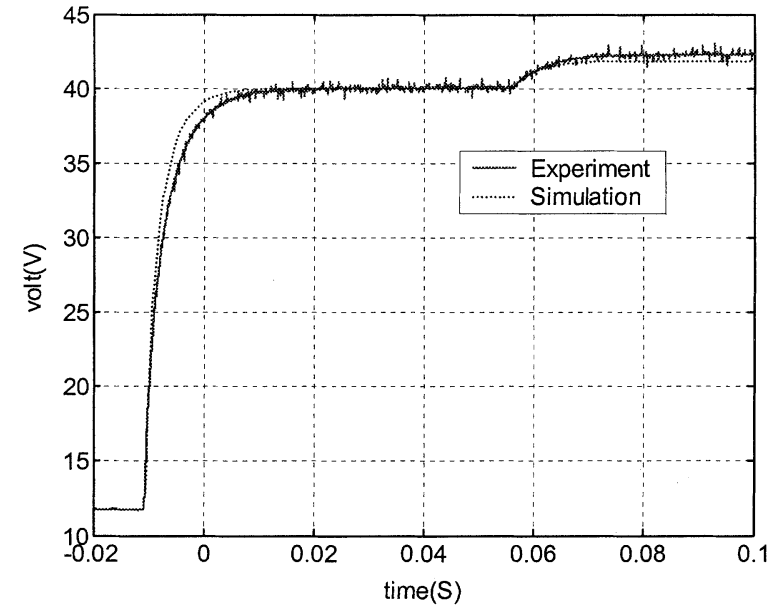

Fig. 15. Output voltage during step-load variation: simulation (dashed line) and experiment (solid line).

control laws. At the beginning the control is not active and the output voltage is equal to the input supply voltage $(12 \mathrm{~V})$ due to the presence of the boost converter diode.

\section{A. Simple Synergetic Control Law}

As a first case we adopt the manifold definition of (10). This case is expected to be the least robust to load variation because (10) uses the current reference, which is kept at the nominal value at all times.

Both in simulation and experiment, we see that the output voltage is disturbed by the step load variation, reaching a new steady-state value that is different from the desired $40-\mathrm{V}$ reference (see Fig. 15). Besides the steady-state error, the system response to the step-load variation is fairly slow and it is desirable to improve it.

\section{B. Addition of Integral Error Term}

This result suggests the need for improvement of our definition for the target manifold. To obtain a zero-steady-state error, control law (21) with an integral voltage error term can be used. The results are shown in Fig. 16. As explained above, this control law gives an overall second-order behavior with overshoot at startup. As a result of the introduction of the integral error term, there is zero steady state output voltage error. However, the system response time to a step-load change is still quite slow.

The input current also shows an interesting behavior. At startup, a 20\% higher current peak is present in the case where the integral term is added.

\section{Control Law With High-Pass-Filtered Inductor Current}

As noted above, one of the reasons for the steady-state output voltage error after the change of the load is the fact that the synergetic control law (10) incorporates the inductor current reference-but that reference cannot be a static quantity when the load is variable. A solution to this problem is to adopt control law (25) that uses a high-pass-filtered measurement of the inductor current and does not need the inductor current reference.

The result is shown in Fig. 17. Notice that the steady-state output voltage error is significantly reduced with respect to the

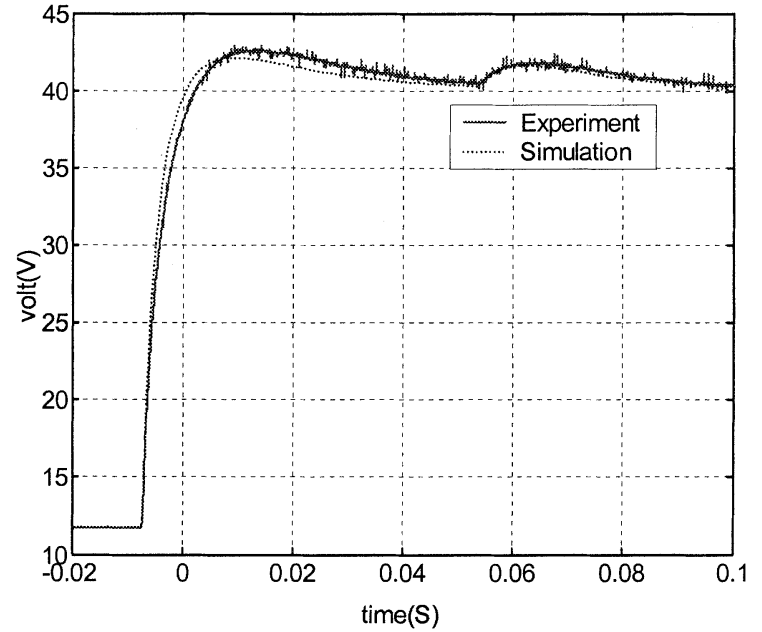

Fig. 16. Output voltage (simulation and experiment) when controller includes integral term.

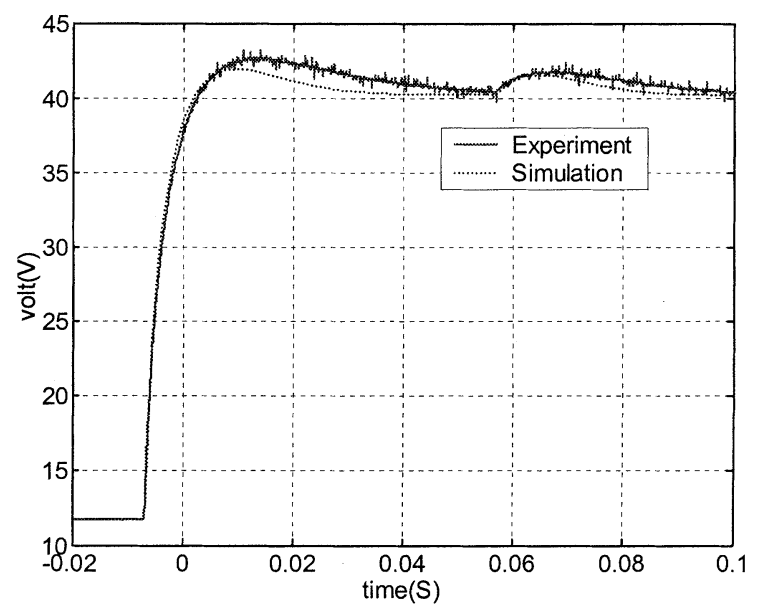

Fig. 17. Output voltage (simulation and experiment) when controller includes high-pass-filtered measured current term.

simple case of Fig. 15. Still, this solution does not improve the response speed.

\section{Adaptation of Control Parameter}

Now, we introduce the control law with adaptive gain $k$ given by (20).

The results obtained with this approach are illustrated in Fig. 18. We see that introduction of the adaptive term has a positive effect on control performance. The startup transient speed is increased and the effect of step load change on the output voltage is so small that it is not discernible with the voltage scale used. This indicates that the steady-state error is reduced with respect to the simple case and the speed of response is significantly improved. Fig. 19 shows the inductor current waveform with the initial overshoot and the response to the step-load change.

\section{DISCUSSION}

At this point, we can reach some conclusions regarding the different control laws used. The simple control law exhibits a slow response time and gives rise to a significant steady-state error. Fig. 20 is a zoomed version of Fig. 15 showing the detail 


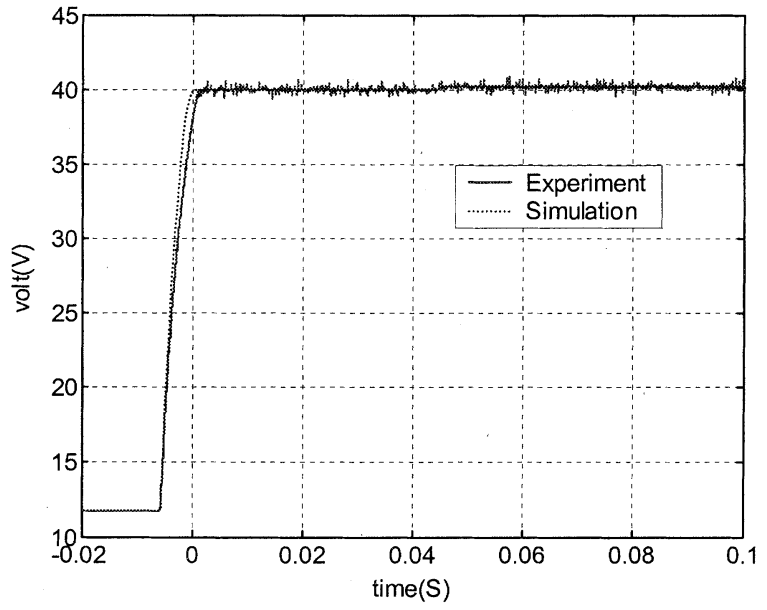

Fig. 18. Output voltage (simulation and experiment) results with adaptive control law.

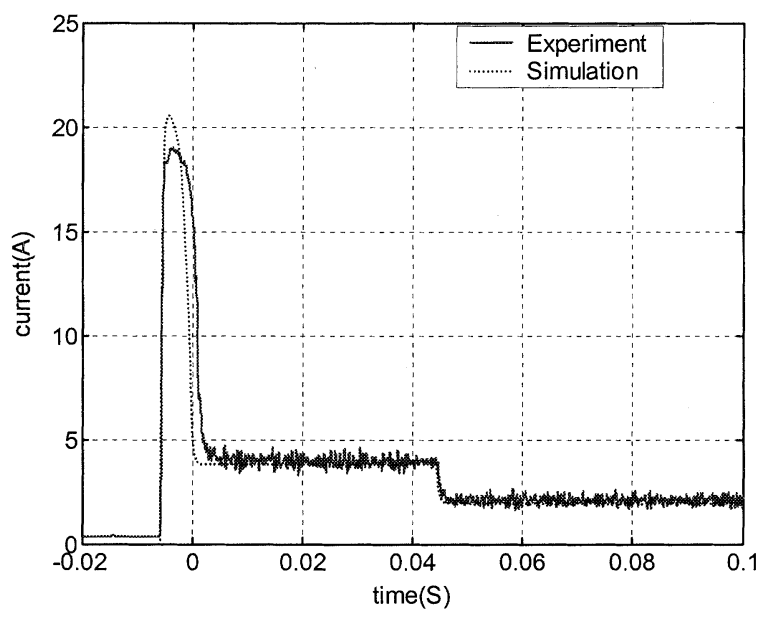

Fig. 19. Inductor current with adaptive control law.

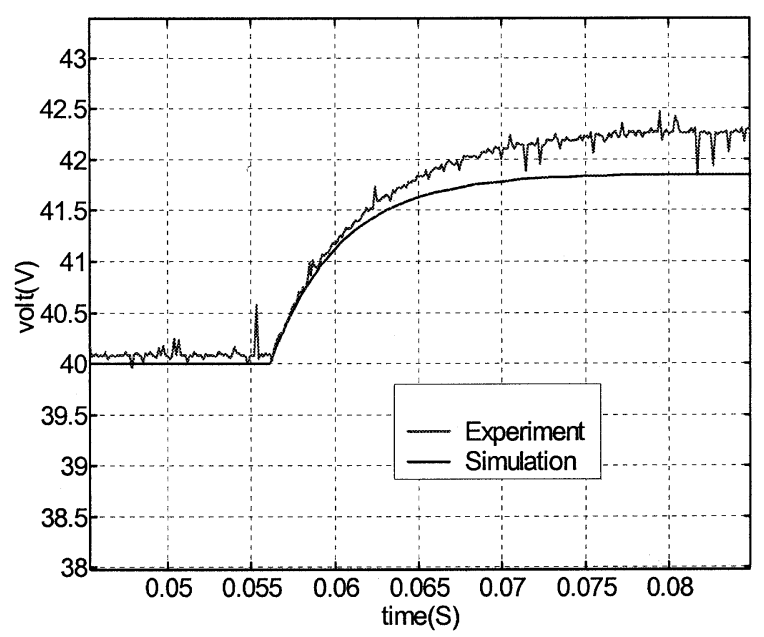

Fig. 20. Step-load response for the simple synergetic control (zoomed version of Fig. 15).

of the step load response. The response time is approximately 12 ms. Analysis using (10) shows that the response time could be reduced by reducing $k$. Unfortunately, simulation shows that the large-signal stability of the system is compromised by a choice of $k$ smaller than 0.5 . The conclusion is that with the simple

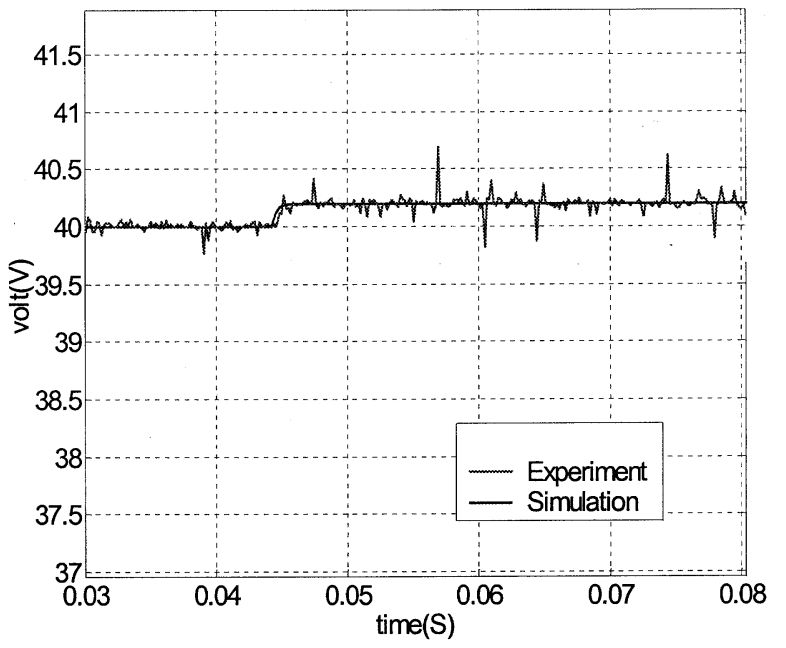

Fig. 21. Step-load response for the adaptive synergetic control (zoomed version of Fig. 18).

control law it is difficult to obtain large-signal stability and fast step-load response.

The addition of an integral term solves the steady-state error problem, but it does not give an improvement in step response speed. The step response speed could be improved by increasing the integral term gain, but this would cause an unacceptable output voltage overshoot at startup. The value used in the experiment already gives a $6 \%$ output voltage overshoot, as shown in Fig. 16.

Similar comments apply to the control law with highpassfiltered inductor current. This control law reduces the steadystate error, but it does not give an improvement in step response speed.

The adaptive control allows the achievement of these goals by reducing $k$ when the converter is close to steady state, guaranteeing fast step load response without compromising largesignal stability. Fig. 21 is a zoomed version of Fig. 18 showing the detail of the step load response. The steady-state error is 0.2 $\mathrm{V}$, which is $0.5 \%$. Notice also that the response time is approximately $0.8 \mathrm{~ms}$, which is 15 times faster than the response with the simple control law.

The response times obtained with the various control laws can be estimated using a small-signal model as explained in [12].

In conclusion, the adaptive control gives the better trade-off between large-signal stability and load step response time.

\section{CONCLUSION}

The basic application of synergetic theory to the control of a boost converter is introduced in [8]. In this paper variations of the basic control law were explored. Among these are the following.

- Two different implementations of a control law that limits inductor current overshoot.

- A control law that includes dynamic adaptation of the control parameter depending on the output voltage error. This control law gives a better tradeoff between transient and steady-state performance.

- A control law that includes an integral error term to eliminate steady-state error. 
- A control law that uses the high-pass-filtered inductor current and does not require knowledge of the reference current, which is load dependent for a desired output voltage.

These control features can also be combined. The different control options demonstrate the flexibility of the synergetic control approach. Simulation and experimental results for all control laws are given under reference step variation and step-load variation. Good agreement between simulation and experimental results is found. The results show that it is possible to synthesize a control law that is highly insensitive to parameter variations. This result is particularly interesting because the independence from model parameters seems to be one of the most important concerns in this kind of control.

\section{REFERENCES}

[1] S. Sanders, J. Noworolski, X. Z. Liu, and G. C. Verghese, "Generalized averaging method for power conversion circuits," IEEE Trans. Power Electron., vol. 6, pp. 251-258, Apr. 1991.

[2] D. M. Mitchell, DC-DC Switching Regulator Analysis. New York: McGraw-Hill, 1988.

[3] R. W. Erickson, S. Cuk, and R. D. Middlebrook, "Large-scale modeling and analysis of switching regulators," Proc. IEEE PESC'82, pp. 240-250, 1982.

[4] P. Maranesi, M. Riva, A. Monti, and A. Rampoldi, "Automatic synthesis of large signal models for power electronic circuits," presented at the IEEE PESC'99, Charleston, SC, July 1999.

[5] V. I. Utkin, "Variable structure system with sliding modes," IEEE Trans. Ind. Electron., vol. AC 22, pp. 212-222, Apr. 1977.

[6] L. Ben-Brahim and A. Kawamura, "Digital control of induction motor current with deadbeat response using predictive state observer," IEEE Trans. Power Electron., vol. 7, pp. 551-559, July 1992.

[7] A. Kolesnikov et al., Modern Applied Control Theory: Synergetic Approach in Control Theory (in Russian). Moscow-Taganrog, Russia: TSURE Press, 2000, vol. 2.

[8] A. Kolesnikov, G. Veselov, A. Kolesnikov, A. Monti, F. Ponci, E. Santi, and R. Dougal, "Synergetic synthesis of Dc-Dc boost converter controllers: Theory and experimental analysis," in Proc. IEEE APEC, Dallas, TX, Mar. 10-14, 2002, pp. 409-415.

[9] K. J. Astrom and B. Wittenmark, Computer-Controlled Systems. Upper Saddle River, NJ: Prentice-Hall, 1997.

[10] R. Dougal, T. Lovett, A. Monti, and E. Santi, "A multilanguage environment for interactive simulation and development of controls for power electronics," presented at the IEEE PESC, Vancouver, BC, Canada, 2001.

[11] A. Monti, E. Santi, R. A. Dougal, and M. Riva, "Rapid prototyping of digital controls for power electronics," IEEE Trans. Power Electron. vol. 18, pp. 915-923, May 2003.

[12] A. Monti, R. Dougal, E. Santi, D. Li, and K. Proddutur, "Compensation for step-load variations when applying synergetic control," presented at the IEEE APEC, Miami Beach, FL, Feb. 9-13, 2003.

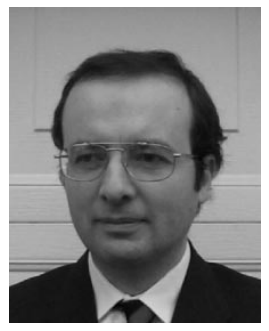

Enrico Santi (S'89-M'94-SM'01) received the Dr. Ing. degree in electrical engineering from the University of Padua, Padua, Italy, in 1988, and the M.S and Ph.D. degrees from California Institute of Technology, Pasadena, in 1989 and 1994, respectively.

He was a Senior Design Engineer with TESLAco from 1993 to 1998 , where he was responsible for the development of various switching power supplies for commercial applications. Since 1998, he has been an Assistant Professor in the Electrical Engineering Department, University of South Carolina, Columbia. He has published several papers on power electronics and modeling and simulation and is the holder of two patents. His research interests include switched-mode power converters, advanced modeling and simulation of power systems, modeling and simulation of semiconductor power devices, and control of power electronics systems.

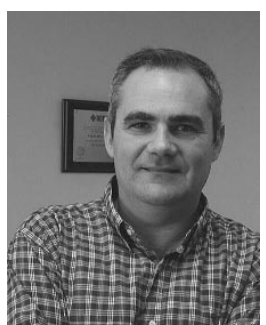

Antonello Monti (M'94-SM'02) received the M.S. degree in electrical engineering and the $\mathrm{Ph} . \mathrm{D}$. degree from the Politecnico di Milano, Milan, Italy, in 1989 and 1994 , respectively.

From 1990 to 1994, he was with the research laboratory of Ansaldo Industria of Milan, where he was responsible for the design of the digital control of a large power cycloconverter drive. From 1995 to 2000 , he was an Assistant Professor in the Department of Electrical Engineering of the Politecnico di Milano. Since August 2000, he has been an Associate Professor in the Department of Electrical Engineering, University of South Carolina, Columbia. He is the author or coauthor of about 120 papers in the areas of power electronics and electrical drives.

Dr. Monti is a Member of the Computers in Power Electronics Committee of the IEEE Power Electronics Society and currently serves as its Chair. In 1998, he served as Chairman of the IEEE Workshop on Computer in Power Electronics held in Como, Italy.

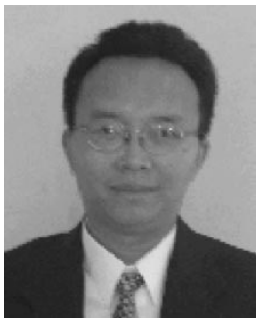

Donghong Li (S'03) received the B.S. degree in electronic science in 1986 from NanKai University, Tianjin, China, and the M.S. degree in computer engineering in 2001 from the University of South Carolina, Columbia, where he is currently working toward the Ph.D. degree in electrical engineering.

His research interests are synergetics and systems synthesis, power electronics, computer engineering, and semiconductor devices and processing.

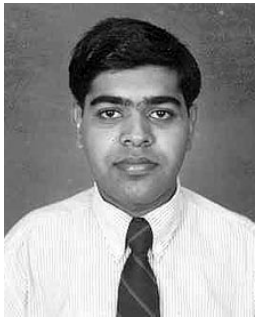

Karthik Proddutur (S'02) received the B.S. degree in electronics and instrumentation engineering from Kakatiya University, Warangal, India, in 2000, and the M.S. degree in electrical engineering from the University of South Carolina, Columbia, in 2002.

$\mathrm{He}$ was a Research Assistant in the Power Electronics Group, Department of Electrical Engineering, University of South Carolina, from 2000 to 2002. His research interests are in the design of control systems for $\mathrm{dc}-\mathrm{dc}$ power electronics applications.

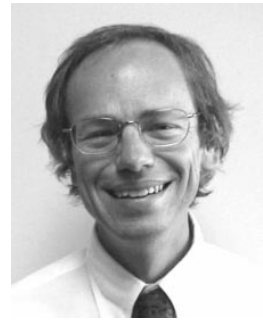

Roger A. Dougal (SM'95) received the Ph.D. degree in electrical engineering from Texas Tech University, Lubbock.

He joined the faculty of the University of South Carolina, Columbia, in 1983. He is Director of the Virtual Test Bed (VTB) project, a multi-disciplinary, multi-university effort to develop a comprehensive simulation and virtual prototyping environment for advanced power systems, integrating power electronics, electromechanics, electrochemistry, and controls into a common test bed. The VTB is unique in allowing the simulation of multi-disciplinary systems by importing models from discipline-specific source languages to a common workspace. In addition to modeling and simulation, his expertise includes power electronics, physical electronics, and power sources.

Dr. Dougal received the Samuel Litman Distinguished Professor of Engineering Award and has been honored as a Carolina Research Professor. 